\title{
Continuum damage mechanics combining thermodynamics with a thoughtful characterization of the microstructure
}

\section{Journal Article}

Author(s):

Hütter, Markus; Tervoort, Theo A. (1)

Publication date:

2008

Permanent link:

https://doi.org/10.3929/ethz-b-000010295

Rights / license:

In Copyright - Non-Commercial Use Permitted

Originally published in:

Acta Mechanica 201(1-4), https://doi.org/10.1007/s00707-008-0064-0 


\title{
M. Hütter · T. A. Tervoort \\ Continuum damage mechanics: combining thermodynamics with a thoughtful characterization of the microstructure
}

\author{
Dedicated to Professor Wilhelm Schneider on the occasion of his 70th birthday
}

Received: 29 January 2008 / Revised: 3 April 2008 / Published online: 9 August 2008

(C) Springer-Verlag 2008

\begin{abstract}
We formulate a macroscopic description of the mechanics of damaged materials. To represent the microstructure, the distribution of crack sizes is captured by way of the Minkowski functionals, or so-called quermass integrals, while a second-rank tensor is used to describe the average orientation of the cracks. A two phase-type approach is adopted to distinguish elastically strained material from unstrained regions in the wake of the cracks. Using nonequilibrium thermodynamic techniques, the driving force for the growth of the microcracks is naturally identified. In particular, Griffith's law is generalized to assemblies of polydisperse crack sizes. Due to the detailed characterization of the microstructure, we are also able to account for the plastic zones at the rims of the cracks that are known to hamper the crack growth, and to discuss possible forms of the damage parameter. The presented approach separates in a transparent fashion the incorporation of fundamental thermodynamic and mechanic principles on one hand, from the specification of the material and details of the crack formation and growth on the other hand.
\end{abstract}

\section{Introduction}

Continuum damage mechanics (CDM) is a relatively new branch of solid mechanics that bridges the fields of continuum mechanics and fracture mechanics. It is an experimental fact that many materials experience micro-crack formation as a prelude to macroscopic fracture, and one of the main objectives of CDM is to capture the essentials of the nucleation and evolution of this micro-damage that often occurs during finite deformation of both ductile and brittle materials.

A concise review of CDM was compiled by Krajcinovic [1]. Within CDM there is a broad distinction between micromechanical models, that consider the detailed stress and strain fields around a microscopic crack, and phenomenological models, that use internal variables to describe the influence of micro-damage on the macroscopic deformation behavior. Focusing on the latter, many internal parameters have been proposed to describe the evolution of damage in materials. Budiansky and O'Connell [2] used a self-consistent procedure to arrive at a crack-density parameter to describe the reduction of the elastic constants that, in the case of an isotropic distribution of penny-shaped cracks, equals the number density of cracks times the average of the cube of the crack radius. Also vectors [3,4], second order tensors [5] and even eighth-order tensors [1] have been used to describe the evolution and orientation distribution of damage in materials. One of the most popular concepts in CDM is based on the notion of a so-called "effective stress" combined with the hypothesis of strain equivalence [6-8]. In this approach, a damage parameter is defined that transforms the nominal stress tensor into an effective stress tensor. For anisotropic damage, this damage parameter is a fourth order tensor that reduces to a scalar in case of isotropic damage. The hypothesis of strain equivalence then states that the effect of damage can be described by applying the constitutive equations for the undamaged material and

M. Hütter $(\varangle) \cdot$ T. A. Tervoort

ETH Zürich, Zurich, Switzerland

E-mail: markus.huetter@math.ethz.ch 
replace the nominal stress tensor with the effective stress tensor. Using these concepts, for isotropic damage in a given cross section through a material point, the damage parameter can be interpreted as the fraction of void area to the total area, or, alternatively, as a loss of compliance, and can be measured simply by comparing the stiffness of undamaged material with that of damaged material upon unloading.

In many theoretical expositions on CDM, the thermodynamics of hidden variables $[9,10]$ has been applied to ensure a correct constitutive description of damage. Typically, the usual balance laws of mass, momentum, moment of momentum and energy, combined with a detailed expression of the Helmholtz free energy as a function of all, including the hidden (damage) variables, are augmented with the Gibbs-Duhem inequality, specifying the non-negative entropy production density $[3,4,8,11,12]$. Applied in this manner, the thermodynamics of hidden variables to describe damage is formally analogous to the more well-known use of hidden variables to describe finite plasticity [13]. However, in most cases, the free energy expression is not used to feed detailed information about crack dimensions on the thermodynamic state of the material, but is merely selected such that it coincides with the effective stress tensor assumption [6-8]. Also the representation theorem of tensor functions [14-16] has been used to arrive at general expressions to describe damage [3,5], but this, unfortunately, often leads to an abundant amount of material coefficients.

In many materials, damage is preceded by plastic deformation. However, most CDM treatments of this so-called "ductile damage" associate damage solely with changes in the elastic properties of the material, assuming additive contributions of damage and plastic deformation to the free energy expression [11,17], although also more elaborate couplings between damage and plastic deformation have been considered [18].

The use of internal ("hidden") variables to characterize the non-equilibrium thermodynamic state of a system, also has a long tradition in the field of polymer rheology. The current most successful constitutive equations that accurately describe the non-linear viscoelastic flow of polymer melts are based on evolution equations for detailed microscopic variables such as the second moment of the end-to-end distance vector of the polymer chains, or the distribution of orientation vectors along the primitive path [19,20]. A general twogenerator formalism, coined "GENERIC" (General Equation for the Nonequilibrium Reversible-Irreversible Coupling), that can be used either in bracket or operator form, was shown to be particularly helpful in guiding a correct description of the non-equilibrium thermodynamics of these complex fluids [21-23]. Recently, GENERIC was successfully applied also in the field of solid mechanics to obtain an Eulerian representation of finite non-isothermal anisotropic elastic and elasto-viscoplastic behavior of compressible solids, using the elastic deformation gradient as structural variable $[24,25]$. It is the objective of this research to apply GENERIC to obtain the full set of dynamic equations that describe continuum damage mechanics in finite, non-isothermal anisotropic brittle materials, based on detailed crack parameters. Particular emphasis will be on the relation between the emerging evolution equations for these crack parameters and Griffith's law [26-28]. Also the implications of coupling between tensorial internal variables on the symmetry of the stress tensor will be discussed.

The manuscript is organized as follows. The GENERIC framework of nonequilibrium thermodynamics is presented in Sect. 2 and applied to formulate the evolution equations for brittle damage mechanics in Sect. 3. Finally, in Sect. 3.6, the model is compared to Griffith's law and other existing models in CDM. The main conclusions are then summarized in Sect. 4.

\section{Thermodynamic formalism}

Nonequilibrium thermodynamics acts as a guard-rail, helping the modeler to cast the understanding of a complex system with internal variables in a form that complies with certain principles of thermodynamics. There is a wide variety of approaches to nonequilibrium thermodynamics modeling, and the relations between many of them have been established $[29,30]$. Here, we choose the general equation for the nonequilibrium reversible-irreversible coupling (GENERIC) framework by Grmela and Öttinger [21-23]. In regard to the topic of this paper, this method seems to be the most suitable one, in particular owing to its large flexibility in using structural variables and to its applicability to nonisothermal situations.

A decade ago, the GENERIC formalism has been developed for describing closed non-equilibrium systems [21-23]. When trying to formulate a model in that framework, the first step is to choose the variables that describe the situation of interest. Similar to the procedure in equilibrium thermodynamics, the choice of variables must be such that they are independent and sufficient to capture the essential physics. Such a set of variables shall here be denoted by $\boldsymbol{x}$. Note that $\boldsymbol{x}$ may have both discrete as well as continuous indices (for field variables). According to GENERIC, the time evolution of the variables $\boldsymbol{x}$ of an isolated system can be 
written in the form

$$
\frac{\partial \boldsymbol{x}}{\partial t}=\boldsymbol{L}(\boldsymbol{x}) \cdot \frac{\delta E}{\delta \boldsymbol{x}}+\boldsymbol{M}(\boldsymbol{x}) \cdot \frac{\delta S}{\delta \boldsymbol{x}},
$$

where the two generators $E$ and $S$ are the total energy and entropy functionals in terms of the state variables $\boldsymbol{x}$, and $\boldsymbol{L}$ and $\boldsymbol{M}$ are certain matrices (operators). The two contributions to the time evolution of $\boldsymbol{x}$ generated by the total energy $E$ and the entropy $S$ are called the reversible and irreversible contributions, respectively. The operator $\boldsymbol{L}$ describes the kinematics, while the operator $\boldsymbol{M}$ accounts for a wealth of irreversible processes, and hence contains material parameters such as diffusion coefficients, viscosities, relaxation times, and reaction constants. The matrix multiplications (symbol .) imply not only summations over discrete indices but may also include integration over continuous variables, in particular for non-local field theories, and $\delta / \delta \boldsymbol{x}$ typically implies functional rather than partial derivatives (for more details see [21-23]).

The GENERIC structure also imposes certain conditions on the building blocks in (1). First, Eq. (1) is supplemented by the mutual degeneracy requirements

$$
\begin{gathered}
\boldsymbol{L}(\boldsymbol{x}) \cdot \frac{\delta S}{\delta \boldsymbol{x}}=\mathbf{0}, \\
\boldsymbol{M}(\boldsymbol{x}) \cdot \frac{\delta E}{\delta \boldsymbol{x}}=\mathbf{0} .
\end{gathered}
$$

The requirement that the functional derivative of the entropy lies in the null-space of $\boldsymbol{L}$ represents the fact that the entropy is not affected by the operator generating the reversible dynamics. Conversely, the energy is conserved by the irreversible contributions, according to (2b). In addition to these degeneracy requirements, $\boldsymbol{L}$ must be anti-symmetric, whereas $\boldsymbol{M}$ needs to be positive-semidefinite and Onsager-Casimir symmetric. As a result of all these conditions one may easily show that the GENERIC equation (1) implies both the conservation of total energy as well as a nonnegative entropy production. Finally, the GENERIC structure requires that the Poisson bracket associated to the operator $\boldsymbol{L}$,

$$
\{A, B\}=\left\langle\frac{\delta A}{\delta \boldsymbol{x}}, \boldsymbol{L} \cdot \frac{\delta B}{\delta \boldsymbol{x}}\right\rangle,
$$

with appropriate scalar product $\langle$,$\rangle , fulfills the Jacobi identity$

$$
\{A,\{B, C\}\}+\{B,\{C, A\}\}+\{C,\{A, B\}\}=0,
$$

for arbitrary functionals $A, B$ and $C$. This identity expresses the time-structure invariance of the reversible dynamics. Given the Poisson bracket (3) together with the antisymmetry and the Jacobi identity (4), the reversible contributions to the evolution equations of the GENERIC take the well-structured form of Hamiltonian dynamics in the geometrical setting of Poisson manifolds.

The complementary degeneracy requirements, the symmetry properties, and the Jacobi identity are essential for formulating proper $\boldsymbol{L}$ - and $\boldsymbol{M}$-matrices when modeling concrete nonequilibrium problems. Various applications have shown that the two-generator idea and the criteria on the two operators have strong implications (e.g., see $[22,23,31,32]$ ).

\section{Dynamic model for crack growth}

\subsection{Variables}

As just discussed, a thoughtful choice of the microstructural variables is of paramount importance. Making such a choice involves the important step of prioritizing versus neglecting features that are thought of as less relevant. Therefore, the final full dynamic model can only be as successful as the starting point was chosen carefully. Keeping this paradigm in mind, we proceed in due detail with the choice of the variables to describe the assembly of microcracks.

In making a choice of variables, we are guided be two opposing requirements. On one hand, certain features of the microstructure must be accounted for in order to give a fair description of the physics. On the other hand, the model should still be tractable, in particular numerical simulations of macroscopic specimens under tensile 

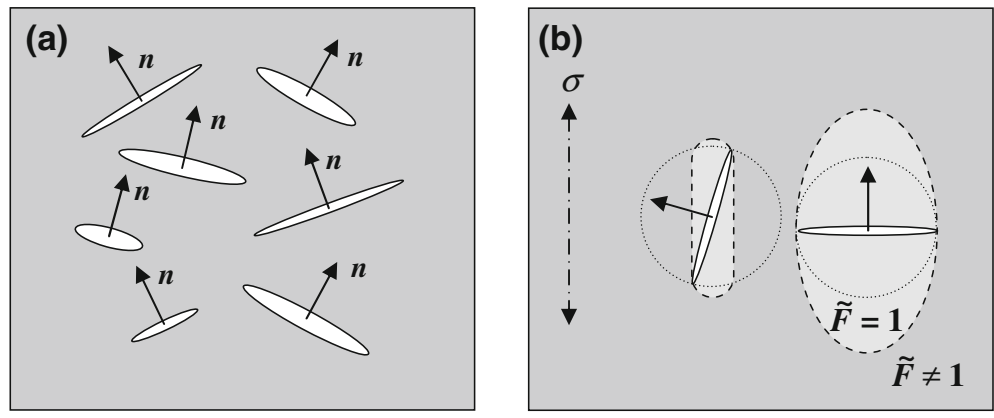

Fig. 1 a Illustration of a random arrangement of flat microcracks with unit normals $\boldsymbol{n}$. Cracks are assumed to have zero volume. The finite thickness of the ellipses indicates that $\boldsymbol{n}$ has a nonvanishing component out of the drawing plane, i.e., the cracks are tilted. b Under a tensile deformation along the direction of the dashed-dotted arrow, regions without elastic strain develop in the wake of the cracks (light grey, bounded by dashed lines). Note that the amount of relaxed material, $\phi=\omega \psi_{3}$, depends on the orientation of the crack with respect to the main tensile direction through $\omega$. The dotted circles indicate the spheres of radius $R$ around each crack as used in the definition of $\psi_{3}$

deformation must be feasible. In this study, we use a continuum formulation with field variables that have a resolution much coarser than the crack size. The cracks are accounted for by measures such as the average crack orientation and the average crack size of all cracks located within one representative volume element, as discussed in the following.

As far as the shape of the cracks is concerned, we restrict our attention to flat, circular cracks of radius $R$, for clarity. If one desires to discuss shapes other than circular disks, the reader is referred to the procedure outlined in [33]. To proceed with the quantification of the microstructure, we point out the following: first, the number density of cracks must be included in order to account adequately for crack initiation. Second, the average radius of cracks is important for quantifying the region around the crack rims that experiences plastic deformation upon crack growth (see Sect. 3.6). Third, the average surface area of cracks is relevant, because in Griffith's treatment of crack growth [26-28] the surface plays a prominent role. And last but not least, if a tensile stress is exerted on the sample, the presence of cracks leads to regions in which the elastic stress is relaxed. Certainly, the size of these regions is related to the size of the cracks, see the light grey domains in Fig. 1b. Therefore, also a volume-type variable must be included in the dynamic model, although the cracks themselves have zero volume. In summary, if the density of crack radii at spatial position $\boldsymbol{r}$ is denoted by $p(R, \boldsymbol{r})$, we propose to use the four variables

$$
\begin{aligned}
& \psi_{0}(\boldsymbol{r})=\int p(R, \boldsymbol{r}) \mathrm{d} R, \\
& \psi_{1}(\boldsymbol{r})=\int R p(R, \boldsymbol{r}) \mathrm{d} R, \\
& \psi_{2}(\boldsymbol{r})=2 \pi \int R^{2} p(R, \boldsymbol{r}) \mathrm{d} R, \\
& \psi_{3}(\boldsymbol{r})=\frac{4 \pi}{3} \int R^{3} p(R, \boldsymbol{r}) \mathrm{d} R,
\end{aligned}
$$

with $\psi_{0}$ the number density of cracks, and $\psi_{1}$ and $\psi_{2}$ the average crack radius and crack surface times the number density of cracks. The quantity $\psi_{3}$ quantifies the volume fraction when associating spheres of radius $R$ with the cracks of radius $R$. The volume fraction of unstrained regions, $\phi$, can then be related to $\psi_{3}$ by

$$
\phi=\omega \psi_{3},
$$

where $0 \leq \omega$ and $\omega$ depends on the loading conditions as illustrated in Fig. 1b. In particular, $\omega=0$ in compression. Note that the four variables in Eqs. (5) are indeed independent since they capture different moments of the distribution of crack radii.

The variables (5) are closely related to the Minkowski functionals, also known as intrinsic volumes (or quermass integrals, curvature integrals), that are used in integral geometry to characterize surfaces and shapes [34-37]. Integral geometric measures in the form of Minkowski functionals find applications in various fields of research, e.g., the study of chemical reaction-diffusion patterns, spinodal decomposition kinetics and the 
structural phase diagrams of emulsions [38,39], as well as to quantify higher order correlations of galaxy cluster distributions in cosmology [40,41] and the heterogeneity of colloidal particle networks [42]. The application of these measures also enters into continuum models for crystallization, assuming that the shape of the crystal phase can be described as an assembly of impinging, randomly oriented and randomly positioned, convex crystals. Quiescent as well as flow-induced crystallization of polymers has been modeled [43-48], and the thermodynamic driving forces for nucleation and growth have been elaborated on $[49,50]$.

The assumption of the cracks being flat allows one to talk in a well defined way about the orientation of the cracks in terms of their surface normals $\boldsymbol{n}$, each of which is unique up to a physically irrelevant sign, see Fig. 1. The orientation of cracks can be modeled in different ways. For example, Muschik et al. [12,51-53] have used the distribution function of the normal vectors as a dynamic variable. However, in view of arriving at a model that is tractable (numerically) also for realistic, macroscopically inhomogeneous situations, we follow a different avenue. In order to represent relevant aspects of the orientation distribution of the cracks in a condensed form, we use as a dynamic variable $\boldsymbol{N}=\langle\boldsymbol{n} \boldsymbol{n}\rangle$ with the property $\operatorname{tr} \boldsymbol{N}=1$, where the average is taken over all cracks within one representative volume element with equal weight to all cracks, regardless of differences in their respective size.

In addition to these variables, to model nonisothermal processes in a moving and compressible material, we use the momentum density $\boldsymbol{u}$, the temperature $T$, and the mass densities of the elastically strained and relaxed material, $\rho_{\mathrm{s}}$ and $\rho_{\mathrm{r}}$. The latter are both so-called "extrinsic" mass densities, i.e., they are given by the ratio of the mass of the respective part divided by the total volume of the representative volume element, and hence they add up to the total mass density of that volume element, $\rho=\rho_{\mathrm{S}}+\rho_{\mathrm{r}}$. The elastic deformation of the material is accounted for by the normalized elastic deformation gradient $\tilde{\boldsymbol{F}}$ with $\operatorname{det} \tilde{\boldsymbol{F}}=1$. For using the $\tilde{\boldsymbol{F}}$ to describe anisotropic finite elasticity and elasto-viscoplasticity in a thermodynamic setting, the reader is referred to [24,25]. In summary, the full set of variables is given by

$$
\boldsymbol{x}=\left(\boldsymbol{u}, T, \rho_{\mathrm{s}}, \rho_{\mathrm{r}},\{\psi\}_{i=0, \ldots, 3}, \boldsymbol{c}, \tilde{\boldsymbol{F}}\right),
$$

which are all functions of the spatial position $\boldsymbol{r}$ and of time $t$. Note that, for technical reasons, we use instead of $N$ an unconstrained tensor $\boldsymbol{c}$, that will later be used to derive the evolution equation of $N$ by way of $N=\boldsymbol{c} / \operatorname{tr} \boldsymbol{c}$.

\subsection{Generating functionals}

The static material properties are specified in terms of the functionals for the total energy and entropy, that according to (1) drive the reversible and irreversible dynamics, respectively. For the time being, we write

$$
\begin{aligned}
& E=\int\left(\frac{\boldsymbol{u}^{2}}{2\left(\rho_{\mathrm{s}}+\rho_{\mathrm{r}}\right)}+e\left(T, \rho_{\mathrm{s}}, \rho_{\mathrm{r}},\{\psi\}_{i=0, \ldots, 3}, \boldsymbol{c} / \operatorname{tr} \boldsymbol{c}, \tilde{\boldsymbol{F}}\right)\right) \mathrm{d}^{3} r, \\
& S=\int s\left(T, \rho_{\mathrm{s}}, \rho_{\mathrm{r}},\{\psi\}_{i=0, \ldots, 3}, \boldsymbol{c} / \operatorname{tr} \boldsymbol{c}, \tilde{\boldsymbol{F}}\right) \mathrm{d}^{3} r
\end{aligned}
$$

where we have only written the kinetic energy term in explicit form. This in turn leads to $\boldsymbol{v}=\delta E / \delta \boldsymbol{u}$ for the velocity field defined by $\boldsymbol{v}=\boldsymbol{u} /\left(\rho_{\mathrm{S}}+\rho_{\mathrm{r}}\right)$. The static thermodynamic properties described by the local energy density $e$ and the entropy density $s$ are still most general, given the set of variables $\boldsymbol{x}$. Specific assumptions about these two functions will only be made in the applications of the general model, see Sect. 3.6. Note that both $e$ and $s$ depend on $c$ only through the combination $N=\boldsymbol{c} / \operatorname{tr} \boldsymbol{c}$ since only the latter is physically significant. In this way, the constraint tr $N=1$ is taken into account properly when performing the functional derivatives $\delta E / \delta \boldsymbol{c}$ and $\delta S / \delta \boldsymbol{c}$ [54]. As the only condition on $e$ and $s$, we require that they be mutually consistent in the sense $T=e_{, T} / s_{, T}$. Throughout the entire manuscript, we use the notation $\left.y(\boldsymbol{x})_{, x_{i}} \equiv\left(\partial y / \partial x_{i}\right)\right|_{x_{k \neq i}}$ for partial derivatives, where $x_{k \neq i}$ denotes the remaining variables in Eq. (7) to be held constant upon differentiation.

\subsection{Reversible dynamics}

The reversible contributions to the time evolution equations of the variables $\boldsymbol{x}$ are given by the Poisson operator $\boldsymbol{L}$. The specific form of the latter can be inferred from the behavior of the variables $\boldsymbol{x}$ under space translations 
[23]. It can be shown that for our present choice of dynamic variables (7) only the momentum column and row of the operator have non-zero entries, namely,

$$
\begin{aligned}
& L_{\alpha \gamma}^{(u u)}=-\nabla_{\gamma} u_{\alpha}-u_{\gamma} \nabla_{\alpha}, \\
& L_{\gamma}^{(T u)}=-\left(\nabla_{\gamma} T\right)-\Omega_{\gamma \mu} \nabla_{\mu}, \\
& L_{\gamma}^{\left(\rho_{\mathrm{s}} u\right)}=-\nabla_{\gamma} \rho_{\mathrm{s}}, \\
& L_{\gamma}^{\left(\rho_{\mathrm{r}} u\right)}=-\nabla_{\gamma} \rho_{\mathrm{r}}, \\
& L_{\gamma}^{\left(\psi_{i} u\right)}=-\nabla_{\gamma} \psi_{i}+a_{i} \psi_{i} \nabla_{\gamma}, \\
& L_{\alpha \beta \gamma}^{(c u)}=-\left(\nabla_{\gamma} c_{\alpha \beta}\right)-c_{\gamma \beta} \nabla_{\alpha}-c_{\alpha \gamma} \nabla_{\beta}, \\
& L_{\alpha \beta \gamma}^{(\tilde{F} u)}=-\left(\nabla_{\gamma} \tilde{F}_{\alpha \beta}\right)+\tilde{F}_{\mu \beta} \nabla_{\mu} \delta_{\alpha \gamma}-\frac{1}{3} \tilde{F}_{\alpha \beta} \nabla_{\gamma}, \\
& L_{\alpha}^{(u T)}=\left(\nabla_{\alpha} T\right)-\nabla_{\mu} \Omega_{\alpha \mu}, \\
& L_{\alpha}^{\left(u \rho_{\mathrm{s}}\right)}=-\rho_{\mathrm{s}} \nabla_{\alpha}, \\
& L_{\alpha}^{\left(u \rho_{\mathrm{r}}\right)}=-\rho_{\mathrm{r}} \nabla_{\alpha}, \\
& L_{\alpha}^{\left(u \psi_{i}\right)}=-\psi_{i} \nabla_{\alpha}+\nabla_{\alpha} a_{i} \psi_{i}, \\
& L_{\alpha \gamma \varepsilon}^{(u c)}=\left(\nabla_{\alpha} c_{\gamma \varepsilon}\right)-\nabla_{\gamma} c_{\alpha \varepsilon}-\nabla_{\varepsilon} c_{\gamma \alpha}, \\
& L_{\alpha \gamma \varepsilon}^{(u \tilde{F})}=\left(\nabla_{\alpha} \tilde{F}_{\gamma \varepsilon}\right)+\nabla_{\mu} \tilde{F}_{\mu \varepsilon} \delta_{\alpha \gamma}-\frac{1}{3} \nabla_{\alpha} \tilde{F}_{\gamma \varepsilon}, \\
& { }_{\alpha}, \ldots, 3,
\end{aligned}
$$

where we assume that the coefficients $a_{i}$ are absolute constants. Subscripts $\gamma$ and $(\gamma, \varepsilon)$ imply contraction with a vector $A_{\gamma}$ and matrix $A_{\gamma \varepsilon}$ multiplied from the right, respectively. All derivative operators act on everything to their right, also on functions multiplied to the right of $\boldsymbol{L}$, except when placed inside of parentheses (...). Since the focus of this manuscript is on the irreversible formation and growth of the microcracks, we refrain from going into any details about the derivation of this expression for the Poisson operator. The interested reader is referred to $[23,24,49]$. We only point out that $\boldsymbol{c}$ is assumed to behave as a lower convected tensor field (in contrast to upper convected) as it can be shown that this leads to the correct contributions in the evolution equation for $\boldsymbol{N}$ for flat disks [55-58], as shown in Sect. 3.5.

The degeneracy condition $\boldsymbol{L} \cdot(\delta S / \delta \boldsymbol{x})=\mathbf{0}$ imposes a condition on the tensor $\boldsymbol{\Omega}$ in the elements $L^{(T u)}$ and $L^{(u T)}$. After some rearrangements one obtains

$$
s_{, T} \boldsymbol{\Omega}=\left(s-\rho_{\mathrm{s}} s_{, \rho_{\mathrm{s}}}-\rho_{\mathrm{r}} s_{, \rho_{\mathrm{r}}}+\sum_{i=0, \ldots, 3}\left(a_{i}-1\right) \psi_{i} s_{, \psi_{i}}\right) \mathbf{1}-2 \boldsymbol{c} \cdot s_{, \boldsymbol{c}}+s_{, \tilde{\boldsymbol{F}}} \cdot \tilde{\boldsymbol{F}}^{T} .
$$

In order to identify the expression for the stress tensor, one needs to write out the momentum balance, i.e. $\left[\partial_{t} \boldsymbol{u}\right]_{\mathrm{rev}}=(\boldsymbol{L} \cdot(\delta E / \delta \boldsymbol{x}))_{\boldsymbol{u}}$, and identify the source term, $\nabla \cdot \boldsymbol{\sigma}^{T}$. Inserting the expression for $\boldsymbol{\Omega}$, using the identity $T=e_{, T} / s_{, T}$, and defining the Helmholtz free energy density as $f=e-T s$, one finds for the stress tensor

$$
\sigma=\sigma^{(\text {iso })}+\sigma^{(N)}+\sigma^{(\tilde{\boldsymbol{F}})},
$$

with

$$
\begin{aligned}
\boldsymbol{\sigma}^{(\text {iso })} & =\left[f-\rho_{\mathrm{s}} f_{, \rho_{\mathrm{s}}}-\rho_{\mathrm{r}} f_{, \rho_{\mathrm{r}}}+\sum_{i=0, \ldots, 3}\left(a_{i}-1\right) \psi_{i} f_{, \psi_{i}}\right] \mathbf{1}, \\
\boldsymbol{\sigma}^{(N)} & =-2 \boldsymbol{c} \cdot f_{, \boldsymbol{c}}, \\
\boldsymbol{\sigma}^{(\tilde{\boldsymbol{F}})} & =f_{, \tilde{\boldsymbol{F}}} \cdot \tilde{\boldsymbol{F}}^{T} .
\end{aligned}
$$


The stress tensor contribution (11d) due elastic deformation is the well known general case for anisotropic finite elasticity $[9,24]$. Since $f_{, \tilde{\boldsymbol{F}}}$ is a constrained derivative due to the condition $\operatorname{det} \tilde{\boldsymbol{F}}=1$, it can be shown that $\operatorname{tr} \sigma^{(\tilde{\boldsymbol{F}})}=0$ (see Appendix). The same holds true for the contribution due to the orientation of cracks, (11c), i.e., $\operatorname{tr} \sigma^{(N)}=0$ as a result of the condition $\operatorname{tr} N=1$ (see Appendix). Therefore, the splitting into isotropic and anisotropic contributions is at the same time a splitting into isotropic bulk and deviatoric, traceless parts.

The Poisson operator $\boldsymbol{L}$ with non-zero elements (9) and (10) is antisymmetric and satisfies the degeneracy condition by construction. In order to prove the Jacobi identity, it is useful to observe that the Jacobi identity is invariant with respect to a transformation of variables from $\boldsymbol{x}$ to $\boldsymbol{x}^{\prime}$ [23]. Since it can be shown that $\left.\partial_{t} s\right|_{\text {rev }}=$ $-\nabla \cdot(s v)$ by the aid of the chain rule and the reversible contributions to the evolution equations given by $\left.\partial_{t} \boldsymbol{x}\right|_{\text {rev }}=\boldsymbol{L} \cdot \delta E / \delta \boldsymbol{x}$ (see also Sect. 3.5), replacing $T$ by $s$ leads to a Poisson operator linear in all field variables. In a straightforward, although lengthy, calculation, the latter Poisson operator can be shown to satisfy the Jacobi identity (see [24] for further details).

\subsection{Irreversible dynamics: formation and growth of microcracks}

In the following, we restrict ourselves to the formation and growth of cracks in solids, i.e., explicit viscoplastic effects and heat conduction are neglected as they have been dealt with elsewhere [23,25]. Since all these effects are of distinct physical origin, such an isolated view on the subproblems is permitted in the absence of cross-couplings, in which case the GENERIC conditions on the friction matrix $\boldsymbol{M}$ are satisfied if they are satisfied for the individual additive contributions. Only in the presence of cross-couplings, not considered here, an integrated treatment of all effects would be required. For the formulation of the formation and growth of cracks (fgc), we proceed along the same lines as in [49], where crystal nucleation and growth have been discussed. In view of the GENERIC equation (1), one must find the generalized friction matrix $\boldsymbol{M}$. As a starting point we write the corresponding contributions to the evolution of the microstructural variables in the form

$$
\left[\partial_{t} \psi_{i}\right]_{(\mathrm{fgc})}=\Upsilon_{i}
$$

which defines the quantities $\Upsilon_{i}$. As a result of these changes in the microstructural variables, also the other variables experience changes that can be written in the form

$$
\begin{aligned}
{\left[\partial_{t} \boldsymbol{u}\right]_{(\mathrm{fgc})} } & =\mathbf{0}, \\
{\left[\partial_{t} T\right]_{(\mathrm{fgc})} } & =\sum_{i=0, \ldots, 3} \hat{T}_{i} \Upsilon_{i}, \\
{\left[\partial_{t} \rho_{\mathrm{s}}\right]_{(\mathrm{fgc})} } & =-\sum_{i=0, \ldots, 3} \hat{\rho}_{i} \Upsilon_{i}, \\
{\left[\partial_{t} \rho_{\mathrm{r}}\right]_{(\mathrm{fgc})} } & =\sum_{i=0, \ldots, 3} \hat{\rho}_{i} \Upsilon_{i}, \\
{\left[\partial_{t} \boldsymbol{c}\right]_{(\mathrm{fgc})} } & =\sum_{i=0, \ldots, 3} \hat{\boldsymbol{c}}_{i} \Upsilon_{i}, \\
{\left[\partial_{t} \tilde{\boldsymbol{F}}\right]_{(\mathrm{fgc})} } & =\sum_{i=0, \ldots, 3} \hat{\boldsymbol{F}}_{i} \Upsilon_{i} .
\end{aligned}
$$

The quantities $\hat{T}_{i}, \hat{\rho}_{i}, \hat{\boldsymbol{c}}_{i}$ and $\hat{\boldsymbol{F}}_{i}$ are in general functions of $\boldsymbol{x}$, and therefore significant physical insight can be modeled upon specifying these functions. Some general statements can be made before that specification. The formulations of (12d) and (12e) ensure conservation of the total mass. Furthermore, in order to comply with the symmetry of $\boldsymbol{c}$ and the condition $\operatorname{det} \tilde{\boldsymbol{F}}=1$, all $\hat{\boldsymbol{c}}_{i}$ must be symmetric and $\operatorname{tr}\left(\tilde{\boldsymbol{F}}^{-1, T} \cdot \hat{\boldsymbol{F}}_{i}\right)=0$. The functions $\hat{T}_{i}$ will be used to respect the conservation of the total energy of the system, as expressed by (2b). It is important to note that in (12b)-(12g) only immediate, direct effects of the formation and growth of cracks are represented. The change of the momentum density as a result of the formation and growth of cracks, for example through changes in $\boldsymbol{c}$ and $\tilde{\boldsymbol{F}}$ that in turn affect the stress tensor, is indirect and therefore $\left[\partial_{t} \boldsymbol{u}\right]_{(\mathrm{fgc})}=\mathbf{0}$. 
For later convenience, Eq. (12) is written in the form $\left[\partial_{t} \boldsymbol{x}\right]_{(\mathrm{fgc})}=\sum_{i=0, \ldots, 3} \boldsymbol{D}_{i} \Upsilon_{i}$, which defines the quantities $\boldsymbol{D}_{i}$ as

$$
\boldsymbol{D}_{i}=\left(\mathbf{0}, \hat{T}_{i},-\hat{\rho}_{i}, \hat{\rho}_{i},\left\{\delta_{i j}\right\}, \hat{\boldsymbol{c}}_{i}, \hat{\boldsymbol{F}}_{i}\right)^{T},
$$

with $\left\{\delta_{i j}\right\}$ representing a nonzero entry only in the $\psi_{i}$-component, which in turn leads to $\boldsymbol{D}_{i}$ being linearly independent. On this basis, it can be shown that the friction matrix must be of the form (see [49] for details)

$$
\boldsymbol{M}=\sum_{i, j=0, \ldots, 3} G_{i j} \boldsymbol{D}_{i} \boldsymbol{D}_{j}^{T}
$$

with a symmetric and positive semi-definite $4 \times 4$-matrix $\boldsymbol{G}$. The degeneracy condition $\boldsymbol{M} \cdot(\delta E / \delta \boldsymbol{x})=\mathbf{0}$ then assumes the form $\boldsymbol{D}_{i} \cdot(\delta E / \delta \boldsymbol{x})=\mathbf{0}(i=0, \ldots, 3)$, leading to the conditions

$$
\hat{T}_{i}=-\frac{1}{e_{, T}}\left(\hat{\rho}_{i}\left(e_{, \rho_{\mathrm{r}}}-e_{, \rho_{\mathrm{s}}}\right)+e_{, \psi_{i}}+\hat{c}_{\alpha \beta, i} e_{, c_{\alpha \beta}}+\hat{F}_{\alpha \beta, i} e_{, F_{\alpha \beta}}\right), \quad i=0, \ldots, 3 .
$$

In turn, one obtains for the partial driving forces $D_{i}$ for the formation of micro-cracks

$$
\begin{aligned}
D_{i} & \equiv \boldsymbol{D}_{i} \cdot(\delta S / \delta \boldsymbol{x}) \\
& =-\frac{1}{T}\left(\hat{\rho}_{i}\left(f_{, \rho_{\mathrm{r}}}-f_{, \rho_{\mathrm{s}}}\right)+f_{, \psi_{i}}+\hat{c}_{\alpha \beta, i} f_{, c_{\alpha \beta}}+\hat{F}_{\alpha \beta, i} f_{, F_{\alpha \beta}}\right), \quad i=0, \ldots, 3,
\end{aligned}
$$

with the free energy density $f$ introduced earlier. The matrix $\boldsymbol{G}$ in (14) determines the kinetics of crack formation and growth as can be seen from the evolutions equations

$$
\left[\partial_{t}\left(\begin{array}{l}
\psi_{0} \\
\psi_{1} \\
\psi_{2} \\
\psi_{3}
\end{array}\right)\right]_{(\mathrm{fgc})}=\boldsymbol{G} \cdot\left(\begin{array}{c}
D_{0} \\
D_{1} \\
D_{2} \\
D_{3}
\end{array}\right)=\left(\begin{array}{c}
\mathcal{N} \\
g_{0} G \psi_{0} \\
g_{1} G \psi_{1} \\
g_{2} G \psi_{2}
\end{array}\right) .
$$

The first equality is a direct consequence of writing the irreversible contributions to the evolution equations in the GENERIC form $\boldsymbol{M} \cdot(\delta S / \delta \boldsymbol{x})$. The second equality illustrates the physical interpretation of the individual contributions with the nucleation and radial growth rates of the cracks denoted by $\mathcal{N}$ and $G$, respectively, similar to Schneider's rate equations in polymer crystallization $[43,44]$. In view of the definitions of $\psi_{i}$ in (5), one finds readily $g_{0}=1, g_{1}=4 \pi$, and $g_{2}=2$ for the geometrical prefactors. Given the general positivity and symmetry conditions on $\boldsymbol{G}$ and in view of the second equality in Eq. (17), it can be shown that $\boldsymbol{G}$ can be put in the form [49]

$$
\boldsymbol{G}=\frac{\hat{G}}{g_{2} \psi_{2}}\left(\begin{array}{c}
Q \\
g_{0} \psi_{0} \\
g_{1} \psi_{1} \\
g_{2} \psi_{2}
\end{array}\right) \otimes\left(\begin{array}{c}
Q \\
g_{0} \psi_{0} \\
g_{1} \psi_{1} \\
g_{2} \psi_{2}
\end{array}\right),
$$

with $\hat{G} \geq 0$ a kinetic prefactor. The only freedom, at least in principle, consists in including a positive additive contribution in the upper left corner of the matrix $\boldsymbol{G}$, that can not be determined within this formalism. Upon setting that additional term to zero, the rank of $\boldsymbol{G}$ drops from two to one, representing the fact that only one physical phenomenon is described. In other words, the formation and growth of cracks have the same thermodynamic driving force. The form (18) can be used in turn to express the nucleation rate and the growth rate as

$$
\begin{aligned}
\mathcal{N} & =\hat{G} Q A, \\
G & =\hat{G} A,
\end{aligned}
$$

with the full thermodynamic driving force

$$
A=\frac{Q}{g_{2} \psi_{2}} D_{0}+\frac{g_{0} \psi_{0}}{g_{2} \psi_{2}} D_{1}+\frac{g_{1} \psi_{1}}{g_{2} \psi_{2}} D_{2}+D_{3}
$$


that will be discussed more explicitly below. The significance of the full driving force can be seen also from the entropy production due to the formation and growth of cracks. Using the chain rule for the entropy density $s(\boldsymbol{x})$ with (12) and the expression $\hat{T}_{i}$ in (15) expressing energy conservation, it can be shown that the change in the entropy density at constant energy density is proportional to the change in the Helmholtz free energy density at constant temperature. This result can be rewritten with the aid of $D_{i}$ defined in (16) to arrive at

$$
\left[\left.\partial_{t} s\right|_{e}\right]_{(\mathrm{fgc})}=-\frac{1}{T}\left[\left.\partial_{t} f\right|_{T}\right]_{(\mathrm{fgc})}=\hat{G} g_{2} \psi_{2} A^{2}
$$

which is quadratic in the full driving force. If one uses the relation $\hat{G} g_{2} \psi_{2} A^{2}=\left[\left.\partial_{t} \psi_{3}\right|_{e}\right]_{(\mathrm{fgc})} A,(21)$ represents a generalization of the entropy production derived by Rice [59,60]. In Sect. 3.6, the relation to Rice's expression is illustrated for a specific example.

The kinetic prefactor $\hat{G}$ is in general a complicated function of the variables in $\boldsymbol{x}$ in order to represent the physics of the kinetics of crack growth. Particularly, two aspects are mentioned here. First, the size of cracks can only remain constant or grow under tension normal to the crack surface, however, the crack size can not decrease upon compression. This fact can be represented in the function $\hat{G}$ if the latter vanishes under conditions that represent a compression in the direction of the average crack surface normal, In other words, $\hat{G}$ depends on both the stress tensor $\sigma$ and the average crack orientation $N$. A second important effect that impacts the kinetics is the plastic deformation that occurs at the crack tip and that hampers crack growth. The implementation of this effect through $\hat{G}$ is discussed in Sect. 3.6.

\subsection{Full set of time-evolution equations}

Collecting the reversible and irreversible contributions to the evolution equations discussed in Sects. 3.3 and 3.4 , one obtains by virtue of the GENERIC equation (1):

$$
\begin{aligned}
\partial_{t} \boldsymbol{u} & =-\nabla \cdot\left(\boldsymbol{v} \boldsymbol{u}-\boldsymbol{\sigma}^{T}\right), \\
\partial_{t} T & =-\boldsymbol{v} \cdot \nabla T-\boldsymbol{\Omega}: \boldsymbol{\kappa}^{T}+\sum_{i=0, \ldots, 3} \hat{T}_{i} \Upsilon_{i}, \\
\partial_{t} \rho_{\mathrm{S}} & =-\nabla \cdot\left(\rho_{\mathrm{s}} \boldsymbol{v}\right)-\sum_{i=0, \ldots, 3} \hat{\rho}_{i} \Upsilon_{i}, \\
\partial_{t} \rho_{\mathrm{r}} & =-\nabla \cdot\left(\rho_{\mathrm{r}} \boldsymbol{v}\right)+\sum_{i=0, \ldots, 3} \hat{\rho}_{i} \Upsilon_{i}, \\
\partial_{t} \psi_{i} & =-\nabla \cdot\left(\psi_{i} \boldsymbol{v}\right)+a_{i} \psi_{i}(\nabla \cdot \boldsymbol{v})+\Upsilon_{i}, \quad i=0, \ldots, 3, \\
\partial_{t} \boldsymbol{c} & =-\boldsymbol{v} \cdot \nabla \boldsymbol{c}-\boldsymbol{\kappa}^{T} \cdot \boldsymbol{c}-\boldsymbol{c} \cdot \boldsymbol{\kappa}+\sum_{i=0, \ldots, 3} \hat{\boldsymbol{c}}_{i} \Upsilon_{i}, \\
\partial_{t} \tilde{\boldsymbol{F}} & =-\boldsymbol{v} \cdot \nabla \tilde{\boldsymbol{F}}+\left(\boldsymbol{\kappa}-\frac{1}{3}(\operatorname{tr} \boldsymbol{\kappa}) \mathbf{1}\right) \cdot \tilde{\boldsymbol{F}}+\sum_{i=0, \ldots, 3} \hat{\boldsymbol{F}}_{i} \Upsilon_{i},
\end{aligned}
$$

with $\boldsymbol{\sigma}$ the stress tensor (11), velocity field $\boldsymbol{v}=\boldsymbol{u} / \rho$, transpose velocity gradient $\boldsymbol{\kappa}=(\nabla \boldsymbol{v})^{T}, \boldsymbol{\Omega}$ and $\hat{T}_{i}$ given by Eqs. (10) and (15), respectively, and

$$
\left(\begin{array}{l}
\Upsilon_{0} \\
\Upsilon_{1} \\
\Upsilon_{2} \\
\Upsilon_{3}
\end{array}\right)=\hat{G} A\left(\begin{array}{c}
Q \\
g_{0} \psi_{0} \\
g_{1} \psi_{1} \\
g_{2} \psi_{2}
\end{array}\right),
$$

with the full driving force $A$ specified in Eq. (20), and geometrical coefficients discussed after Eq. (17). 
Let us recall that the tensor $\boldsymbol{c}$ was introduced for technical reasons only, while our physical interest was in the orientation tensor $N=c / \operatorname{tr} c$. Using the chain rule and (22f), the evolution equation for $N$ can be derived,

$$
\partial_{t} N=-v \cdot \nabla N-\kappa^{T} \cdot N-N \cdot \kappa+N N:\left(\kappa+\kappa^{T}\right)+\sum_{i=0, \ldots, 3}\left(\frac{\hat{c}_{i}}{\operatorname{tr} c}-N \frac{\operatorname{tr} \hat{c}_{i}}{\operatorname{tr} c}\right) \Upsilon_{i} .
$$

The terms on the right hand side proportional to the velocity gradient are well known for flat disks with the approximation of Doi, in which the exact expression $\boldsymbol{\kappa}:\langle\boldsymbol{n n n n \boldsymbol { n }}\rangle$ is replaced by $\boldsymbol{\kappa}:\langle\boldsymbol{n n}\rangle\langle\boldsymbol{n n}\rangle$ [55-58]. In order to arrive at a closed set of evolution equations using the variable $N$, without any explicit occurrence of $c$, it can be shown that the following conditions must be satisfied. First, the functions for the energy density $e$ and entropy density $s$ must depend on $\boldsymbol{c}$ only through the quantity $\boldsymbol{c} / \operatorname{tr} \boldsymbol{c}$, which has already been implemented in (8). This leads to the stress tensor $\boldsymbol{\sigma}$ in Eq. (11) and $\boldsymbol{\Omega}$ given by Eq. (10) being dependent on $\boldsymbol{c}$ only through $\boldsymbol{N}$. As a second requirement, also $\hat{\rho}_{i}, \hat{\boldsymbol{F}}_{i}$, and $\hat{\boldsymbol{c}}_{i} / \operatorname{tr} \boldsymbol{c}$ must depend on $\boldsymbol{c}$ only through $\boldsymbol{N}$, which in turn also leads to that desirable property for $\hat{T}_{i}$ given by Eq. (15).

\subsection{Discussion}

\subsubsection{Relation to Griffith's law for crack growth}

The advantage of the above procedure consists in formulating a closed set of thermodynamically admissible evolution equations, without ever relying on a specific form of the generating functionals, i.e., without restriction to special materials. In this section, we proceed to making specific ansatzes for the energy density $e$ and entropy density $s$ used in the generating functionals (8), in order to provide an illustrative example. In addition, important physical insight can be accounted for by appropriate choices for $\hat{\rho}_{i}$ and, in particular, for $\hat{\boldsymbol{c}}_{i}$ and $\hat{\boldsymbol{F}}_{i}$. The ramifications in (22) of all these specifications is then discussed.

We make an ansatz for the Helmholtz free energy, from which both $e$ and $s$ can be derived by way of $-s=f_{, T}$ and $e=f+T s$,

$$
f=f_{\mathrm{s}}+f_{\mathrm{r}}+f_{\mathrm{i}} .
$$

This free energy expression contains a bulk contribution $f_{\mathrm{s}}$ that represents the strained material, a bulk contribution $f_{\mathrm{r}}$ that represents the relaxed material, and an interface contribution $f_{\mathrm{i}}$, for which we make the ansatz

$$
\begin{aligned}
& f_{\mathrm{s}}=(1-\phi) \mathcal{F}\left(T, \rho_{\mathrm{s}} /(1-\phi), \tilde{\boldsymbol{F}}, \boldsymbol{c}\right), \\
& f_{\mathrm{r}}=\phi \mathcal{F}\left(T, \rho_{\mathrm{r}} / \phi, \tilde{\boldsymbol{F}}=\mathbf{1}, \boldsymbol{c}\right), \\
& f_{\mathrm{i}}=\psi_{2} \gamma(T),
\end{aligned}
$$

with $\gamma$ the surface tension, and $\phi=\omega \psi_{3}$ the volume fraction of elastically unstrained material, introduced in Eq. (6). While $\omega$ is in principle a general function of $\boldsymbol{x}$, we make the simplifying assumption that, to first order, the change in $\omega$ due to the formation and growth of cracks is negligible. In turn, this means that in the calculation of the full driving force $A, \omega$ can be considered to be a constant. Note that we have used the identical function $\mathcal{F}$ in both $f_{\mathrm{s}}$ and $f_{\mathrm{r}}$, which is the Helmholtz free energy per unit volume of the respective "phase", since both the elastically strained and relaxed regions represent the same material. In addition to the thermodynamic properties (25), we also specify

$$
\begin{aligned}
\hat{\rho}_{i}=0, & i=0, \ldots, 2, \\
\hat{\boldsymbol{c}}_{i}=\mathbf{0}, & i=1, \ldots, 3, \\
\hat{\boldsymbol{F}}_{i}=\mathbf{0}, & i=0, \ldots, 3,
\end{aligned}
$$

i.e., mass is transferred between the relaxed and the strained regions only by way of a change in $\psi_{3}$. As far as the rate of change in $\rho_{\mathrm{r}}$ versus change in $\phi$ is concerned, $\hat{\rho}_{3} / \omega$, its value must lie between the mass densities of strained and relaxed material with respect to their respective volumes, $\rho_{\mathrm{s}} /(1-\phi)$ and $\rho_{\mathrm{r}} / \phi$, which however, are also approximately equal, i.e.,

$$
\frac{\rho_{\mathrm{s}}}{1-\phi} \simeq \frac{\rho_{\mathrm{r}}}{\phi} \simeq \frac{\hat{\rho}_{3}}{\omega}
$$


In Eqs. (26) it is further assumed that crack growth does not have an immediate, first order effect on the elastic strain $\tilde{\boldsymbol{F}}$ in the strained material. Furthermore, Eq. (26b) states that the orientation of existing cracks does not change as an immediate consequence of crack growth. However, $\hat{\boldsymbol{c}}_{0}$ may well be nonzero since the formation of new cracks occurs with a preferred orientation that is related to the applied elastic strain. As a consequence, the average orientation of all cracks, namely $\boldsymbol{N}=\langle\boldsymbol{n n}\rangle$ that includes both grown and freshly nucleated cracks, experiences a distortion. Under these assumptions, the partial driving forces (16) can be calculated, which in turn lead to

$$
A=\frac{1}{T}\left[\omega(\mathcal{F}(\tilde{\boldsymbol{F}})-\mathcal{F}(\mathbf{1}))-\frac{g_{1} \psi_{1}}{g_{2} \psi_{2}} \gamma-\frac{Q}{g_{2} \psi_{2}} \hat{c}_{\alpha \beta, 0} f_{, c_{\alpha \beta}}\right]
$$

for the full driving force $A$ for crack formation and growth (20).

Let us first interpret this expression for $Q=0$, i.e., in the absence of crack formation. This situation is representative of the pre-existence of small defects in the material, which will grow into finite-size cracks under appropriate stress conditions. The driving force shows a size-dependence through the ratio $\psi_{1} / \psi_{2}$. Furthermore, the prefactor $\omega$ in front of the bulk terms illustrates the effect of the orientation of the cracks with respect to the strain direction. If the crack normal is parallel to the main loading (tensile) direction, the bulk contribution to the driving force is large. However, if the crack normal is oriented perpendicular the bulk contribution becomes small and is over-compensated by the surface term. Therefore, the relation (28) for $Q=0$ is a generalization of Griffith's law for crack growth in a twofold sense. First, it accounts for an assembly of many cracks that are polydisperse in size. And second, it explicitly incorporates the orientation dependence in a condensed fashion. With this form of $A$, the relation of (21) to the entropy production as derived by Rice $[59,60]$ becomes clear, where the bulk term in (28) is closely related to the so-called Irwin energy release rate [61].

In the presence of crack nucleation, $Q \neq 0$, an additional contribution to the driving force occurs due to the anisotropic orientation of new cracks which leads to a change in the free energy. This effect can be discussed by an analogy. In the case of crystallization, the change in the thermodynamic potential by a small virtual transfer of mass, i.e., the difference in chemical potentials, enters into the driving force for the phase transformation in such a way that the (free) energy of the system is lowered upon phase transformation. Similarly, in the deformed system with cracks, the driving force is affected by a potential gain/loss in the free energy upon changing the average orientational of the cracks by crack formation.

\subsubsection{Plastic deformation at the rim of cracks}

It is well known that the growth of cracks is hampered by plastic deformation around the crack tip [26,27]. For cracks of spherical shape as studied in this contribution, the spatial region of plastic deformation is a ring torus with radius from the center of the hole to the center of the torus tube $R+R_{\mathrm{p}}$, and the radius of the tube denoted by $R_{\mathrm{p}}$ (see Fig. 2). If we assume that $R_{\mathrm{p}}$ does not depend significantly on the crack radius, the volume fraction of tori is given by

$$
\phi_{\mathrm{t}}=2 \pi^{2} R_{\mathrm{p}}^{2} \psi_{1}
$$

It must be noted that this relation is valid even for a polydisperse size distribution of cracks, since $\psi_{1}$ correctly captures the first moment of the crack size distribution. In other words, only due to the careful characterization of the crack morphology it becomes possible to quantify the volume that undergoes plastic deformation at the crack tips.

Since the plastic deformation dissipates energy and hence hampers crack growth, a first order approximation to include this effect in our model consists in making the kinetic factor $\hat{G}$ in Eq. (23) depend on $\phi_{\mathrm{t}}$ in an appropriate way. Another, more advanced option is the following. The model discussed so far is a twocomponent model in the sense that only relaxed and strained domains have been distinguished. This description can be augmented by including a third component, representing the plastically deformed rim around the cracks.

Doing so requires the introduction of another deformation gradient, similar to $\tilde{\boldsymbol{F}}$, which corresponding evolution equations that include the plastic deformation. For clarity, we leave this extension for future work. 


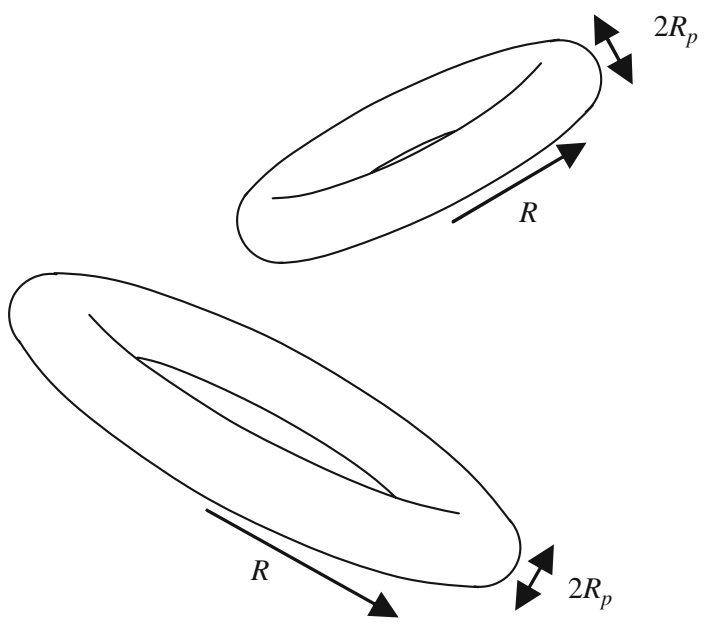

Fig. 2 Illustration of the ring torus, in which plastic deformation occurs upon crack growth, for two cracks of different size $R$. Although the cracks have different radius, $R$, the thickness $2 R_{\mathrm{p}}$ of the torus is independent of $R$

\subsubsection{Damage parameter}

The damage parameter is in general introduced to relate mechanical properties of the damaged and undamaged material. This being said, there are different definitions of the damage parameter. In the following, we briefly discuss two of the most prominent definitions in view of our modeling approach.

A common definition of a damage parameter is given as follows. After putting a test plane with surface normal $\boldsymbol{m}$ through the damaged material, one measures the fraction of the "effective resisting area", usually denoted by $D$ [6-8]. If the distribution of cracks is anisotropic, the quantity $D$ depends on the orientation of the test plane, i.e., on $\boldsymbol{m}$. In order to account for anisotropy in the crack orientation when relating the stress $\sigma$ and the "effective stress" $\overline{\boldsymbol{\sigma}}$, a fourth rank tensor $\mathcal{M}^{-1}$ is introduced [8],

$$
\bar{\sigma}_{\alpha \beta}=\mathcal{M}_{\alpha \beta \gamma \varepsilon}^{-1}: \sigma_{\gamma \varepsilon}
$$

where $\mathcal{M}^{-1}$ depends on the anisotropic orientation distribution and on the size of the cracks. Due to our detailed description of the microstructure, such effects can be accounted for in the following manner. In terms of our variables, $\psi_{2} /\left(2 \psi_{0}\right)$ is the average surface area of a crack. In order to discuss failure of the material, this quantity is to be related to the square of the average distance between cracks, i.e., to $\psi_{0}^{-2 / 3}$. Therefore, a definition equivalent to $D$ discussed above consists in our setting of $D=\psi_{2} \psi_{0}^{-1 / 3} / 2$. Obviously, the critical value of this parameter for the onset of material failure depends on the average orientation of the cracks with respect to the main stress components. To express that fact, the tensor $\mathcal{M}_{\alpha \beta \gamma \varepsilon}^{-1}$ may be expressed in the form

$$
\mathcal{M}_{\alpha \beta \gamma \varepsilon}^{-1}=p\left(D / D_{c_{1}}\right) N_{\alpha \gamma} N_{\beta \varepsilon}+p\left(D / D_{c_{2}}\right)\left(\delta_{\alpha \gamma}-N_{\alpha \gamma}\right)\left(\delta_{\beta \varepsilon}-N_{\beta \varepsilon}\right) .
$$

Doing so allows one to account for different critical values $D_{c_{1}}$ and $D_{c_{2}}$ of $D$ depending on the relative orientation between cracks and main stress components. In analogy to [8], the function $p$ can be taken of the form $p(z)=(1-z)^{-1}$.

A crucial point must be made in this discussion of damage parameters. While usually the damaged and undamaged material are related on the basis of moduli or the stress tensor, in our approach the thermodynamic potentials $e$ and $s$ in (8) play the key role. Only through these two functions are we allowed to incorporate our physical understanding of the thermodynamic and mechanical behavior of the material, while the GENERIC treatment merely shows the ramifications of these specifications in the constitutive relations and the evolution equations. Although this may seem as a limitation of our approach on the first sight, it comes with the invaluable benefit of respecting fundamental principles of mechanics and thermodynamics. In particular, the damage parameter $D=\psi_{2} \psi_{0}^{-1 / 3} / 2$ enters directly into $e$ and $s$, possibly in an anisotropic manner in combination with $N$. This in turn does not only affect the stress tensor (11), but also the driving force for the formation and growth of cracks (20). For illustration of that point, we briefly discuss the stress tensor expression based on 
the free energy density (25), that has been introduced earlier to examine the driving force $A$. The ramification of (25) for the elastic contribution (11d) to the stress tensor is given by

$$
\boldsymbol{\sigma}^{(\tilde{\boldsymbol{F}})}=f_{, \tilde{\boldsymbol{F}}} \cdot \tilde{\boldsymbol{F}}^{T}=(1-\phi) \mathcal{F}(\tilde{\boldsymbol{F}})_{, \tilde{\boldsymbol{F}}} \cdot \tilde{\boldsymbol{F}}^{T}+f_{, \phi} \psi_{3} \omega_{, \tilde{\boldsymbol{F}}} \cdot \tilde{\boldsymbol{F}}^{T}
$$

where we have assumed that the elastic stress in regions with $\tilde{\boldsymbol{F}}=\mathbf{1}$ vanishes. In Eq. (32), the term that derives from the dependence of $\omega$ on the state of deformation $\tilde{\boldsymbol{F}}$ is usually not considered explicitly. If that term is neglected, we get to interpret $\phi$ as a damage parameter since $1-\phi$ relates the stress $\sigma^{(\tilde{\boldsymbol{F}})}$ to what is commonly termed "effective stress", i.e., to $\mathcal{F}(\tilde{\boldsymbol{F}})_{, \tilde{\boldsymbol{F}}} \cdot \tilde{\boldsymbol{F}}^{T}$. Such identification of the damage parameter is in line with [2], where the effective moduli of the damaged material are related to the moduli of the undamaged material. For an isotropic orientation distribution of circular cracks, the structural variable that enters into this relation according to [2] is proportional to the variable $\psi_{3}$ in our description.

\subsubsection{Symmetry of stress tensor}

While the isotropic contribution to the stress tensor (11a) is obviously symmetric, the symmetry of the other two contributions, $\sigma^{(N)}$ and $\sigma^{(\tilde{\boldsymbol{F}})}$, must be discussed carefully. To analyze the problem, it is illustrative to decompose the free energy into three additive contributions, one depending on $\boldsymbol{c}$ only (through $\boldsymbol{N}$ ), another depending on $\tilde{\boldsymbol{F}}$ only, and a third coupling term that depends both on $\boldsymbol{c}$ and $\tilde{\boldsymbol{F}}$,

$$
f=f_{1}(\boldsymbol{N})+f_{2}(\tilde{\boldsymbol{F}})+f_{3}(\boldsymbol{N}, \tilde{\boldsymbol{F}}) .
$$

Since $f_{1}$ can only depend on the orientation through the invariants of $N$, it is straightforward to show that the corresponding contribution to $\boldsymbol{\sigma}^{(\boldsymbol{N})}$ is symmetric. The effect of $f_{2}$ on $\boldsymbol{\sigma}^{(\tilde{\boldsymbol{F}})}$ can be discussed as in general finite elasticity. In particular, the symmetry of $\sigma^{(\tilde{\boldsymbol{F}})}$ is guaranteed since the free energy depends on $\tilde{\boldsymbol{F}}$ only through the (constrained) right Cauchy-Green strain tensor, $\tilde{\boldsymbol{C}}=\tilde{\boldsymbol{F}}^{T} \cdot \tilde{\boldsymbol{F}}[9,24]$.

The effect of $f_{1}$ and $f_{2}$ being discussed, we can concentrate in the following on the mixed contribution in (33), i.e., $f_{3}$. We point out that such a nontrivial coupling term occurs, e.g., in the ansatz (25), in which $\phi=\omega \psi_{3}$ in (6) depends on both $\boldsymbol{c}$ and $\tilde{\boldsymbol{F}}$ by way of $\omega$. One can show that if $f_{3}$ assumes the form $f_{3}(\boldsymbol{N}, \tilde{\boldsymbol{F}})=$ $f_{3}^{\prime}(\boldsymbol{N}) f_{3}^{\prime \prime}(\tilde{\boldsymbol{F}})$ with two scalar-valued functions $f^{\prime}$ and $f^{\prime \prime}$, the corresponding contribution to the stress tensor will be symmetric, the argument being identical to the ones for $f_{1}$ and $f_{2}$ above. Therefore, we only need to consider cases for $f_{3}$ in which $\boldsymbol{N}$ and $\tilde{\boldsymbol{F}}$ are coupled tensorially. Physically reasonable ansatzes for such tensorial coupling must account for the following fact. The left and right index of the deformation gradient $\tilde{\boldsymbol{F}}$ refer to the current (deformed) and reference (undeformed) state, respectively [9,24]. Since $N$ describes the orientation of the cracks in the deformed state, $N$ must be contracted only with the left index of $\tilde{\boldsymbol{F}}$. In the following paragraph, two specific couplings of this type are considered.

As a first example of proper coupling between $\boldsymbol{N}$ and $\tilde{\boldsymbol{F}}$, we study the case in which $f_{3}$ depends on $\boldsymbol{N}$ and $\tilde{\boldsymbol{F}}$ only through the (symmetric) tensorial quantity $\boldsymbol{A}=\tilde{\boldsymbol{F}}^{T} \cdot \boldsymbol{N} \cdot \tilde{\boldsymbol{F}}$. Using the chain rule, one can show that the corresponding contributions (11c) and (11d) in the stress tensor read

$$
\begin{aligned}
& \boldsymbol{\sigma}_{\left[f_{3}\right]}^{(\boldsymbol{N})}=-2\left[\boldsymbol{N} \cdot \tilde{\boldsymbol{F}} \cdot \frac{\partial f_{3}}{\partial \boldsymbol{A}} \cdot \tilde{\boldsymbol{F}}^{T}-\operatorname{tr}\left(\boldsymbol{N} \cdot \tilde{\boldsymbol{F}} \cdot \frac{\partial f_{3}}{\partial \boldsymbol{A}} \cdot \tilde{\boldsymbol{F}}^{T}\right) \boldsymbol{N}\right], \\
& \boldsymbol{\sigma}_{\left[f_{3}\right]}^{(\tilde{\boldsymbol{F}})}=2\left[\boldsymbol{N} \cdot \tilde{\boldsymbol{F}} \cdot \frac{\partial f_{3}}{\partial \boldsymbol{A}} \cdot \tilde{\boldsymbol{F}}^{T}-\frac{1}{3} \operatorname{tr}\left(\boldsymbol{N} \cdot \tilde{\boldsymbol{F}} \cdot \frac{\partial f_{3}}{\partial \boldsymbol{A}} \cdot \tilde{\boldsymbol{F}}^{T}\right) \mathbf{1}\right] .
\end{aligned}
$$

One observes immediately that the first, potentially nonsymmetric, terms in Eqs. (34a) and (34b) cancel out in the full stress tensor (11a), while the second terms in Eqs. (34a) and (34b) are manifestly symmetric. As a second example, we consider a function $f_{3}$ that depends on $\boldsymbol{N}$ and $\tilde{\boldsymbol{F}}$ only through $\boldsymbol{A}^{\prime}=\tilde{\boldsymbol{F}}^{T} \cdot \boldsymbol{N} \cdot \boldsymbol{N} \cdot \tilde{\boldsymbol{F}}$. For this case, the corresponding contributions in the stress tensor assume the form

$$
\begin{aligned}
& \boldsymbol{\sigma}_{\left[f_{3}\right]}^{(\boldsymbol{N})}=-2\left[\boldsymbol{N} \cdot \boldsymbol{N} \cdot \tilde{\boldsymbol{F}} \cdot \frac{\partial f_{3}}{\partial \boldsymbol{A}^{\prime}} \cdot \tilde{\boldsymbol{F}}^{T}+\boldsymbol{N} \cdot \tilde{\boldsymbol{F}} \cdot \frac{\partial f_{3}}{\partial \boldsymbol{A}^{\prime}} \cdot \tilde{\boldsymbol{F}}^{T} \cdot \boldsymbol{N}\right]+4 \operatorname{tr}\left(\boldsymbol{N} \cdot \tilde{\boldsymbol{F}} \cdot \frac{\partial f_{3}}{\partial \boldsymbol{A}^{\prime}} \cdot \tilde{\boldsymbol{F}}^{T} \cdot \boldsymbol{N}\right) \boldsymbol{N} \\
& \boldsymbol{\sigma}_{\left[f_{3}\right]}^{(\tilde{\boldsymbol{F}})}=+2\left[\boldsymbol{N} \cdot \boldsymbol{N} \cdot \tilde{\boldsymbol{F}} \cdot \frac{\partial f_{3}}{\partial \boldsymbol{A}^{\prime}} \cdot \tilde{\boldsymbol{F}}^{T}-\frac{1}{3} \operatorname{tr}\left(\boldsymbol{N} \cdot \boldsymbol{N} \cdot \tilde{\boldsymbol{F}} \cdot \frac{\partial f_{3}}{\partial \boldsymbol{A}^{\prime}} \cdot \tilde{\boldsymbol{F}}^{T}\right) \mathbf{1}\right]
\end{aligned}
$$


which again leads to a total stress tensor (11a) that is manifestly symmetric. Obviously, a coupling in terms of both $\boldsymbol{A}$ and $\boldsymbol{A}^{\prime}$, i.e. $f_{3}(\boldsymbol{N}, \tilde{\boldsymbol{F}})=\tilde{f}_{3}\left(\boldsymbol{A}, \boldsymbol{A}^{\prime}\right)$, also leads to a symmetric stress tensor (11a).

For more general expressions for the coupling term $f_{3}$ in the free energy, the reader is referred to the literature on the representation theorem of tensor functions [14-16]. It may be seen as part of our model that the form of the $(\boldsymbol{N}, \tilde{\boldsymbol{F}})$-coupling must guarantee the symmetry of the stress tensor (11). If a free energy expression is used that leads to antisymmetric contributions in the stress tensor, all possible ramifications must be explored carefully, e.g., the conservation of the angular momentum.

\section{Summary}

Using GENERIC, a general formalism for non-equilibrium thermodynamics, a new set of dynamic equations for the continuum description of microscopic damage during non-isothermal finite deformation in anisotropic brittle materials is presented. As internal variables to describe microscopic cracks, the first four moments of the distribution function for crack radii and the second moment of the orientation distribution function of crack normal vectors are selected. The state of elastic deformation is captured by the elastic part of the deformation gradient. The dynamic equations describing the evolution of damage during deformation are depicted in an Eulerian setting, and an expression of the stress tensor is obtained, that details the contributions of cracks due to their number density and size distribution as well as due to their orientation.

Assuming that the Helmholtz free energy of the system consists of three contributions, related to strained and unstrained material and the surface energy of the cracks, respectively, a driving force for crack growth emerges that is a generalization of Griffith's law. In addition, it is shown that this assumption for the Helmholtz free energy leads to an elastic contribution of the stress tensor that is reminiscent to an "effective stress tensor" approach, utilizing the Budiansky-O'Connell damage parameter.

Alternatively, the framework presented here with its detailed description of the microstructure is also capable of capturing damage parameters that are commonly based on the concept of the effective resisting area, even for anisotropic materials.

Special attention is given to allowable couplings between the two tensorial parameters that are involved in the constitutive description of the damaged material, the elastic deformation gradient and the second moment of the orientation distribution function of crack normal vectors, to ensure symmetry of the stress tensor.

The benefit of the presented modeling efforts are a general, thermodynamically correct, approach to continuum damage mechanics, based on a detailed microscopic description of crack nucleation and growth, that can be extended in a straightforward manner to include cross-effects between damage processes and plastic deformation.

\section{Appendix: Constrained derivatives and stress tensor expression}

If the free energy density $f$ depends on the tensor $c$ only through $N=\boldsymbol{c} / \operatorname{tr} \boldsymbol{c}$, the partial derivative can be written as

$$
f_{, c_{\alpha \beta}}=\left\{f_{, N_{\gamma \varepsilon}}\right\} \frac{\partial N_{\gamma \varepsilon}}{\partial c_{\alpha \beta}}=\left\{f_{, N_{\gamma \varepsilon}}\right\}\left(\frac{1}{\operatorname{tr} c} \delta_{\alpha \gamma} \delta_{\beta \varepsilon}-\frac{c_{\gamma \varepsilon}}{(\operatorname{tr} c)^{2}} \delta_{\alpha \beta}\right)
$$

where the expression in the brackets $\{\ldots\}$ is understood as an unconstrained derivative. It follows immediately that the stress tensor contribution $\sigma^{(N)}$ defined in Eq. (11c) is traceless.

If the free energy density $f$ depends on the constrained deformation gradient $\tilde{\boldsymbol{F}}=\boldsymbol{F} / \sqrt[3]{\operatorname{det} \boldsymbol{F}}$, and with the aid of the chain rule and $(\partial \operatorname{det} \boldsymbol{F} / \partial \boldsymbol{F})=(\operatorname{det} \boldsymbol{F})\left(\boldsymbol{F}^{T}\right)^{-1}$, the partial derivative can be written as

$$
f_{, \tilde{F}_{\alpha \beta}}=\left\{f_{, \tilde{F}_{\gamma \varepsilon}}\right\} \sqrt[3]{\operatorname{det} \boldsymbol{F}} \frac{\partial \tilde{F}_{\gamma \varepsilon}}{\partial F_{\alpha \beta}}=\left\{f_{, \tilde{F}_{\gamma \varepsilon}}\right\}\left(\delta_{\alpha \gamma} \delta_{\beta \varepsilon}-\frac{1}{3} F_{\gamma \varepsilon} F_{\beta \alpha}^{-1}\right)
$$

where the expression in the brackets $\{\ldots\}$ is understood as an unconstrained derivative. It follows immediately that the stress tensor contribution $\boldsymbol{\sigma}^{(\tilde{\boldsymbol{F}})}$ defined in Eq. (11d) is traceless. 


\section{References}

1. Krajcinovic, D.: Damage mechanics. Mech. Mater. 8, 117-197 (1989)

2. Budiansky, B., O'Connell, R.J.: Elastic moduli of a cracked solid. Int. J. Solids Struct. 12, 81-97 (1976)

3. Davison, A.L., Stevens, L.: Thermomechanical constitution of spalling elastic bodies. J. Appl. Phys. 44, 668-674 (1973)

4. Krajcinovic, D., Fonseka, G.U.: The continuous damage theory of brittle materials. Part 1: General theory. J. Appl. Mech. 48, 809-815 (1981)

5. Kachanov, M.: Continuum model of medium with cracks. J. Eng. Mech. Div. ASCE 106(EM5), 1039-1051 (1980)

6. Lemaitre, J.: How to use damage mechanics? Nucl. Eng. Des. 80, 233-245 (1984)

7. Lemaitre, J.: A continuous damage mechanics model for ductile fracture. J. Eng. Mater. Techn. (Trans. ASME) 107, 83-89 (1985)

8. Simo, J.C., Ju, J.W.: Strain- and stress-based continuum damage models. 1. Formulation. Int. J. Solids Struct. 23, 821-840 (1987)

9. Truesdell, C., Noll, W.: The Non-Linear Field Theories of Mechanics, 2nd edn. Springer, Berlin (1992)

10. Muschik, W., Papenfuss, C., Ehrentraut, H.: A sketch of continuum thermodynamics. J. Non-Newtonian Fluid Mech. 96, 255-290 (2001)

11. Baker, G., Borst, R.de : An anisotropic thermomechanical damage model for concrete at transient elavated temperatures. Phil. Trans. R. Soc. A 363, 2603-2628 (2005)

12. Papenfuss, C., Ván, P., Muschik, W.: Mesoscopic theory of microcracks. Arch. Mech. 55, 459-477 (2003)

13. Besseling, J.F., van der Giessen, E.: Mathematical modelling of inelastic deformation. In: Knobs, R.J., Morton, K.W. (eds.) Series: Applied Mathematics and Mathematical Computation, vol. 5, Chapman \& Hall, London (1994)

14. Wang, C.C.: A new representation theorem for isotropic functions: An answer to Professor G.F. Smith's criticism of my paper on 'Representations for isotropic functions. 1. Scalar-valued isotropic functions'. Arch. Rat. Mech. Anal. 36, 166-197 (1970)

15. Wang, C.C.: A new representation theorem for isotropic functions: An answer to Professor G.F. Smith's criticism of my paper on 'Representations for isotropic functions. 2. Vector-valued isotropic functions, symmetric tensor-valued isotropic functions, and skew-symmetric tensor-valued isotropic functions'. Arch. Rat. Mech. Anal. 36, 198-223 (1970)

16. Spencer, A.J.M.: Part III. Theory of invariants, pp. 239-353. In: Eringen, A.C. (ed.) Continuum Physics, vol. 1. Mathematics Academic Press, New York (1971)

17. Ju, J.W.: On energy-based coupled elastoplastic damage theories: Constitutive modeling and computational aspects. Int. J. Solids Struct. 25, 803-833 (1989)

18. Hansen, N.R., Schreyer, H.L.: A thermodynamically consistent framework for theories of elastoplasticity coupled with damage. Int. J. Solids Struct. 31, 359-389 (1994)

19. Bird, R.B., Curtiss, C.F., Armstrong, R.C., Hassager, O.: Dynamics of Polymeric Liquids, vol. 2. Kinetic Theory, 2nd edn. Wiley, New York (1987)

20. Larson, R.G.: Constitutive Equations for Polymer Melts and Solutions. Butterworth Publishers, Stoneham (1988)

21. Grmela, M., Öttinger, H.C.: Dynamics and thermodynamics of complex fluids. I. Development of a general formalism. Phys. Rev. E 56, 6620-6632 (1997)

22. Öttinger, H.C., Grmela, M.: Dynamics and thermodynamics of complex fluids. II. Illustrations of a general formalism. Phys. Rev. E 56, 6633-6655 (1997)

23. Öttinger, H.C.: Beyond Equilibrium Thermodynamics. Wiley, Hobroken (2005)

24. Hütter, M., Tervoort, T. A.: Finite anisotropic elasticity and material frame indifference from a nonequilibrium thermodynamics perspective. J. Non-Newtonian Fluid Mech. (special issue IWNET2006) 152, 45-52 (2008)

25. Hütter, M., Tervoort, T. A.: Thermodynamic considerations on nonisothermal finite anisotropic elasto-viscoplasticity. J. Non-Newtonian Fluid Mech. (special issue IWNET2006) 152, 53-65 (2008)

26. Anderson, T.L.: Fracture Mechanics: Fundamentals and Applications, 2nd edn. CRC Press, Boca Raton (1995)

27. Janssen, M., Zuidema, J., Wanhill, R.J.H.: Fracture Mechanics, 2nd edn. DUP Blue Print, Delft (2002)

28. Gdoutos, E.E.: Fracture Mechanics: An Introduction, 2nd edn. (Vol. 123 of Solid Mechanics and Its Applications). Springer, Dordrecht (2005)

29. Grmela, M.: Workshop report. J. Non-Newtonian Fluid. Mech. 69, 105-107 (1997)

30. Jongschaap, R.J.J., Öttinger, H.C.: Workshop report. J. Non-Newtonian Fluid. Mech. 96, 1-3 (2001)

31. Edwards, B.J., Öttinger, H.C.: Time-structure invariance criteria for closure approximations. Phys. Rev. E 56, 4097-4103 (1997)

32. Öttinger, H.C.: Modeling complex fluids with a tensor and a scalar as structural variables. Rev. Mex. Fis. 48(Suppl.1), 220-229 (2002)

33. Hütter, M., Rutledge, G.C., Armstrong, R.C.: Crystal shapes and crystallization in continuum modeling. Phys. Fluids 17, 014107 (2005)

34. Hadwiger, H.: Vorlesungen über Inhalt, Oberfläche und Isoperimetrie. Springer, Heidelberg (1957)

35. Santalò, L.A.: Integral Geometry and Geometric Probability. Addison-Wesley, Reading (1976)

36. Schneider, R.: Convex Bodies: The Brunn-Minkowski Theory. Cambridge University Press, Cambridge (1993)

37. Weil, W.: Stereology: a survey for geometers. In: Gruber, P.M., Wills, J.M. (eds.) Convexity and Its Applications, pp. 360-412. Birkhäuser, Basel (1983)

38. Mecke, K.R.: Integral geometry in statistical physics. Int. J. Mod. Phys. B 12, 861-899 (1998)

39. Mecke, K.R.: Additivity, convexity, and beyond: Applications of Minkowski functionals in statistical physics. In: Mecke, K.R., Stoyan, D. (eds.) Statistical Physics and Spatial Statistics: The Art of Analyzing and Modeling Spatial Structures and Pattern Formation. Vol. 554 of Lecture Notes in Physics, pp. 111-184. Springer, Berlin (2000)

40. Mecke, K.R., Buchert, T., Wagner, H.: Robust morphological measures for large-scale structure in the universe. Astron. Astrophys. 288, 697-704 (1994) 
41. Kerscher, M., Mecke, K.R., Schuecker, P., Böhringer, H., Guzzo, L., Collins, C.A., Schindler, S., De Grandi, S., Cruddace, R.: Non-Gaussian morphology on large scales: Minkowski functionals of the REFLEX cluster catalogue. Astron. Astrophys. 377, 1-16 (2001)

42. Hütter, M.: Heterogeneity of colloidal particle networks analyzed by means of Minkowski functionals. Phys. Rev. E 68, $031404(2003)$

43. Schneider, W., Köppl, A., Berger, J.: Non-isothermal crystallization of polymers. Part 1. System of rate equations. Int. Polym Process 3, 151-154 (1988)

44. Schneider, W., Berger, J., Köppl, A.: Non-isothermal crystallization of polymers: Application of rate equations. In: Güçeri, S.I. (ed.) Proceedings of the 1st International Conference Transport Phenomena in Processing, pp. 1043-1054. Technomic Publ. Co., Lancaster (1992)

45. Eder, G., Janeschitz-Kriegl, H., Liedauer, S.: Crystallization processes in quiescent and moving polymer melts under heat transfer conditions. Prog. Polym. Sci. 15, 629-714 (1990)

46. Eder, G., Janeschitz-Kriegl, H.: Crystallization. In: Meijer, H.E.H. (ed.) Processing of Polymers, vol. 18 of Material Science and Technology, pp. 269-342. Wiley, Weinheim (1997)

47. Zuidema, H.: Flow induced crystallization of polymers. Eindhoven University of Technology, Ph.D. thesis. Eindhoven, The Netherlands (2000)

48. Zuidema, H., Peters, G.W.M., Meijer, H.E.H.: Development and validation of a recoverable strain-based model for flowinduced crystallization of polymers. Macromol. Theor. Simul. 10, 447-460 (2001)

49. Hütter, M.: Thermodynamically consistent incorporation of the Schneider rate equations into two-phase models. Phys. Rev. E 64, 011209 (2001)

50. Hütter, M.: Solidification in closed systems: Cluster size distribution and its driving force. Multiscale Model. Sim. 1, 371$390(2003)$

51. Ván, P., Papenfuss, C., Muschik, W.: Mesoscopic dynamics of microcracks. Phys. Rev. E 62, 6206-6215 (2000)

52. Ván, P., Papenfuss, C., Muschik, W.: Griffith cracks in the mesoscopic microcrack theory. J. Phys. A Math. Gen. 37, 5315$5328(2004)$

53. Papenfuss, C., Böhme, T., Herrmann, H., Muschik, W., Verhás, J.: Dynamics of the size and orientation distribution of microcracks and evolution of macroscopic damage parameters. J. Non-Equil. Thermodyn. 32, 129-142 (2007)

54. Beris, A.N., Edwards, B.J.: Thermodynamics of Flowing Systems with Internal Microstructure. Oxford University Press, Oxford (1994)

55. Larson, R.G.: The Structure and Rheology of Complex Fluids. Oxford University Press, New York (1999)

56. Hinch, E.J., Leal, L.G.: Constitutive equations in suspension mechanics. Part 2. Approximate forms for a suspension of rigid particles affected by Brownian rotations. J. Fluid. Mech. 76, 187-208 (1976)

57. Kröger, M.: Models for Polymeric and Anisotropic Liquids. Lecture Notes in Physics, Vol. 675. Springer, Berlin (2005)

58. Kröger, M., Ammar, A., Chinesta, F.: Consistent closure schemes for statistical models of anisotropic fluids. J. NonNewtonian Fluid Mech. 149, 40-55 (2008)

59. Rice, J.R.: Continuum mechanics and thermodynamics of plasticity in relation to microscale deformation mechanisms. In: Argon, A.S. (ed.) Constitutive Equations in Plasticity, pp. 23-79. MIT Press, Cambridge (1975)

60. Rice, J.R.: Thermodynamics of the quasi-static growth of Griffith cracks. J. Mech. Phys. Solids 26, 61-78 (1978)

61. Irwin, G.R.: Analysis of stresses and strains near the end of a crack traversing a plate. J. Appl. Mech. 24, 361-364 (1957) 